\title{
Una mirada a la Educación Ubicua
}

\section{(A review of Ubiquitous Learning)}

\author{
Carmen Inés Báez Pérez \\ Universidad de Boyacá (Colombia) \\ Clifton Eduardo Clunie Beaufond \\ Universidad Tecnológica de Panamá (Panamá)
}

DOI: $\underline{\text { http://dx.doi.org/10.5944/ried.22.1.22422 }}$

\section{Como referenciar este artículo:}

Báez, C. I. P., y Clunie, B. C. E. (2019). Una mirada a la Educación Ubicua. RIED. Revista Iberoamericana de Educación a Distancia, 22(1), pp. 325-344. doi: http://dx.doi.org/10.5944/ried.22.1.22422

\section{Resumen}

La incorporación de las Tecnologías de la información y la comunicación en el campo de la educación ha permitido grandes cambios en la forma tanto de enseñar como de aprender. Dicha incorporación ha llevado a generar nuevos escenarios en los cuales estos procesos se desarrollan de mejor manera. La educación Ubicua surge de la evolución de las Tecnologías de la Información y la Comunicación (TIC) y su incorporación dentro de los procesos de enseñanza-aprendizaje, buscando acercar el aprendizaje a contextos cercanos a los aprendices, logrando así una formación basada en el contexto. El artículo presenta un estado de arte acerca de la identificación de cuatro aspectos principales que deben ser tenidos en cuenta para la adecuada inclusión de la educación ubicua en un proceso de formación. Los cuatro aspectos identificados son: áreas de conocimiento en las cuales se puede aplicar, niveles educativos en los que se puede implementar, tecnologías de información que facilitan la implementación de recursos digitales para realizar este tipo de aprendizaje y se mencionan modelos de aprendizaje que intervienen en el aprendizaje ubicuo. Estos aspectos se abarcan desde la perspectiva de autores que han trabajado sobre los mismos, dando una visión de los aspectos que se deben considerar para una adecuada incorporación de la educación ubicua en un contexto adecuado. Al finalizar el artículo, se presenta mediante un mapa mental un contexto generalizado de lo expuesto y las conclusiones que se obtienen del desarrollo del mismo.

Palabras clave: actividades de aprendizaje; ambientes de educación ubicua; tendencias en educación; tecnologías de la información y la comunicación. 


\begin{abstract}
The incorporation of Information, Communication and Technologies in the field of education has allowed for great changes in both teaching and learning. This incorporation has led to the generation of new scenarios in which these processes are developed. Ubiquitous education arises from the evolution of Information Technology and its incorporation into the teaching-learning processes, seeking to bring learning closer to the learners, thus achieving a context-based education. This paper is a state of the art presentation of the identification of four main aspects that must be taken into account, for the adequate inclusion of ubiquitous education in a training process. The four aspects identified here are: areas of knowledge in which it can be applied, educational levels in which it can be implemented, information technologies that facilitate the implementation of digital resources to carry out this type of learning, and learning models that intervene in ubiquitous learning These aspects are covered from the perspective of authors who have worked on them, providing a perspective of the aspects that should be considered for an adequate incorporation of ubiquitous education in an appropriate context. Finally, a generalized context of what has been presented and the conclusions obtained from its development are presented through a mental map.
\end{abstract}

Keywords: learning activities; ubiquitous learning environments; education trends; information and communication technology.

La evolución de las Tecnologías de la Información y la Comunicación (de aquí en adelante, TIC) y, particularmente, la incorporación de los dispositivos electrónicos de cómputo móviles, a saber, portátiles, tabletas, teléfonos inteligentes, entre otros; en la vida cotidiana, han generado grandes cambios en la forma que se interrelacionan las personas y el desarrollo de sus actividades, lo cual ha permeado áreas tan diversas como: salud, financiera, ambiental, industria, entre otras.

Una de las áreas, en las cuales se han generado grandes cambios, es en la educación. La incorporación de TIC en el ámbito educativo se hace cada vez más necesaria, dado que las nuevas generaciones han incorporado la tecnología de manera casi natural, generando nuevos retos y propiciando nuevos escenarios en donde su uso sea un mecanismo de apoyo al proceso de adquisición de nuevo conocimiento, bien sea en un proceso formal o en un proceso informal. Según Gros (2015), "Las tecnologías móviles permiten a los estudiantes de todas las edades operar a través de diferentes contextos" (p. 61), así como los estudiantes son capaces de adaptarse a contextos propios, lo cual conduce a experimentar una mejor experiencia de aprendizaje (Kinshuk, 2015).

El reto que ha planteado el uso de las TIC como apoyo a los procesos de enseñanzaaprendizaje ha generado la necesidad de crear nuevas formas de realizarlos, lo cual obliga a realizar cambios en la forma de educar. Es por eso que, desde hace algunos años, se habla de educación tradicional (se desarrolla en un aula de clase), educación a distancia (se desarrolla de forma no presencial parte del tiempo y se realiza 
mediante el uso de tecnologías como televisión, radio y material impreso de apoyo), educación en línea (se realiza mediante el uso de un computador y con el apoyo de una plataforma computacional), luego aparece el concepto de $m$-learning (busca apoyar el proceso de aprendizaje mediante el uso de dispositivos computacionales móviles), este concepto de m-learning ha ido evolucionando hasta lo que hoy en día se conoce como U-learning (ubiquitous learning - Educación ubicua) (Yahya, Arniza y Jalil, 2010), entendiéndose este concepto como "la formación ubicua integra el aprendizaje y la tecnología ubicua dentro de una estrategia formativa y uno de sus frutos más conocidos es el M-learning (utilización de dispositivos móviles para el aprendizaje)" (Fidalgo, 2013, p. 2 )

De acuerdo con Cantillo Valero (2012), "las tecnologías móviles han redibujado el panorama educativo, aportando a la educación no sólo movilidad sino también conectividad, ubicuidad y permanencia, características propias de los dispositivos móviles tan necesarias en los sistemas de educación a distancia” (p. 3), así como también lo afirma Zapata-Ros (2012): "La tecnología ubícua permite a los individuos aprender allí donde estén, y contar para ello con los componentes de su entorno social" (p. 1).

La evolución general del proceso de aprendizaje, desde el punto de vista del ambiente en el cual se desarrollan, se puede visualizar en la figura 1.

Figura 1. Evolución del proceso de aprendizaje

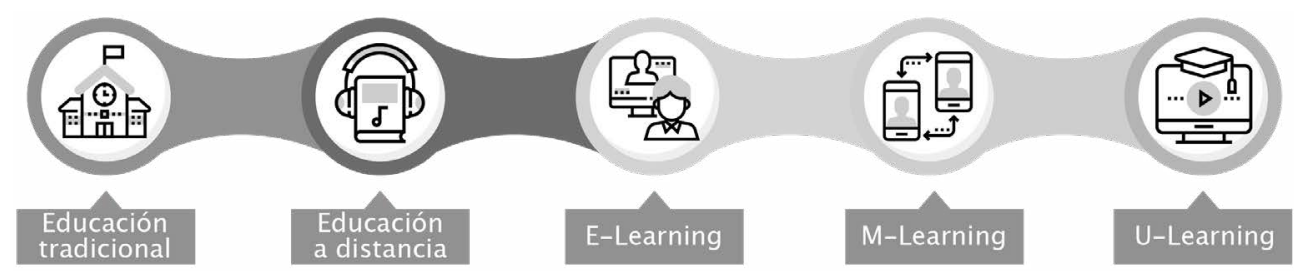

Partiendo de una definición de aprendizaje ubicuo como: "en cualquier lugar, en cualquier momento" (Burbules, 2014, p. 1) y de acuerdo con Barragán Sánchez, Mimbrero Mallado \& Pacheco González-Piñal (2013), "U-learning no sólo implica la posibilidad de aprendizaje en cualquier momento y lugar sino que además integra al concepto de e-learning y m-learning e incluye en la formación a través de la televisión interactiva, mp3 o la Web 2.o" (p. 11), Chi (2012) adiciona que puede realizarse por medio de dispositivos móviles y aplicaciones para dichos dispositivos (p. 1), Burbules (2012) define que el aprendizaje puede darse de la interacción de pares y expertos (p. 7); según Carmona y Puertas (2012) "el U-learning tiene como objetivo crear un ambiente de aprendizaje donde el estudiante esté totalmente inmerso y dónde no sólo adquiere conocimiento sino que también lo comparte con sus compañeros y/o su organización” (p. 25). 
Dado lo anterior, se puede llegar a plantear una definición del concepto como: la educación ubicua permite el aprendizaje en cualquier momento y en cualquier lugar, dependiendo del entorno de aplicación y apoyado por herramientas digitales que permiten la inclusión de diferentes actores dentro del proceso de formación.

Las definiciones anteriores permiten proponer en la figura 2 las características y aspectos principales que involucran el concepto de U-learning.

Figura 2. Definición de U-learning

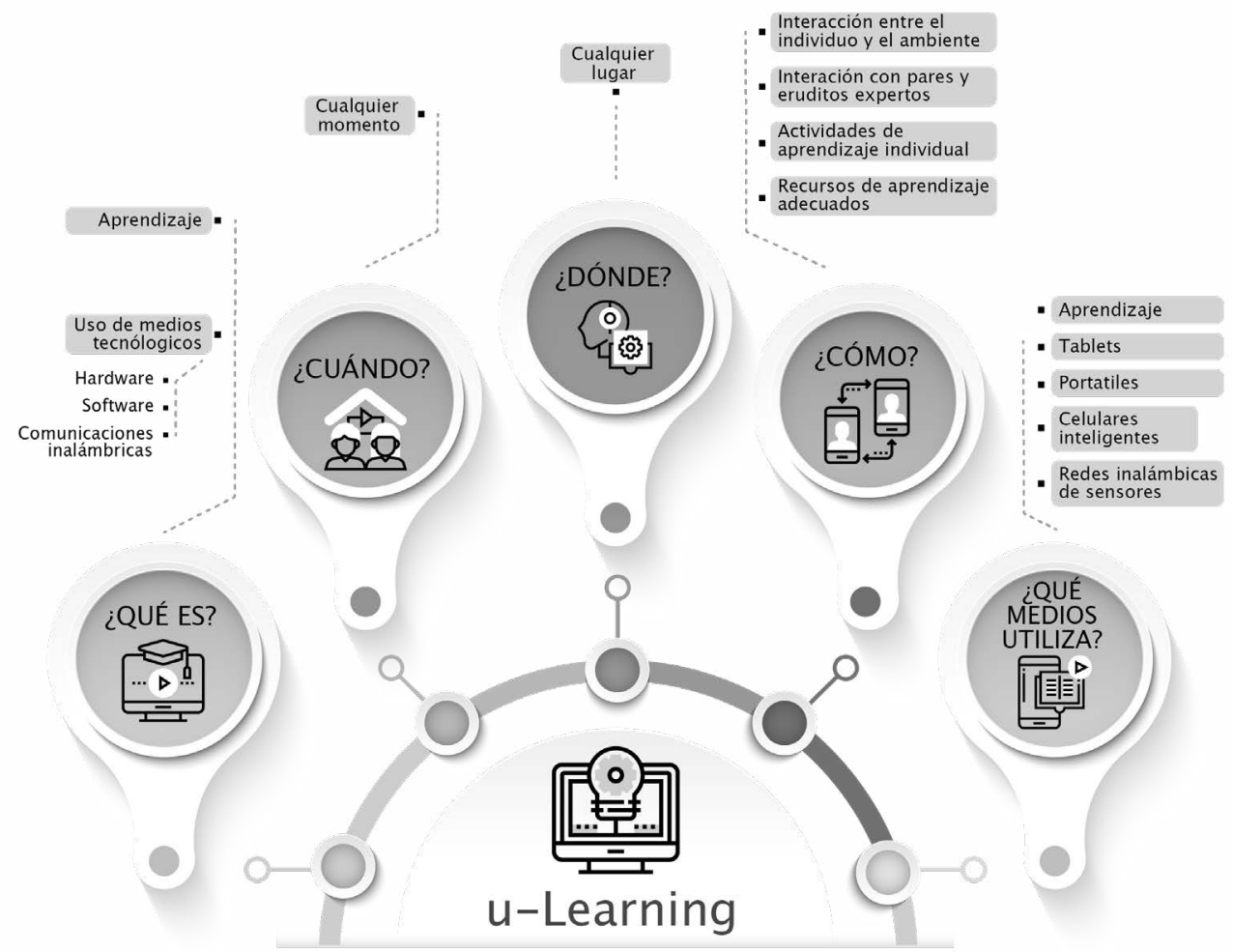

En conclusión, U-learning es un proceso de aprendizaje contextual mediado por tecnología (dispositivos electrónicos móviles de cómputo, redes inalámbricas de comunicaciones, redes de sensores, aplicaciones de software desarrolladas específicamente para ser mediadoras del proceso) independientemente del tiempo y el lugar específico, donde interactúan de manera síncrona/asíncronas redes de estudiantes, docentes y expertos. 
Por otra parte, es importante tener en cuenta que el objetivo final de todo proceso de aprendizaje es mejorar el desempeño y aumentar las capacidades de los estudiantes, dependiendo del objeto de estudio, por lo cual se hace necesario "fomentar la implicación efectiva del alumnado en su propio proceso de aprendizaje, aprovechando el atractivo y el acceso masivo a los smartphones y los dispositivos móviles en general" (Fundación Telefónica, 2014, p. 4), sin olvidar que la calidad debe ser una constante en estos nuevos tipos de proceso de aprendizaje (Zapata-Ros, 2015).

El objetivo de esta revisión bibliográfica es presentar al lector una síntesis de diferentes aspectos que pueden abarcarse y ser tenidos en cuenta a la hora de implementar un proceso de formación ubicua. El enfoque principal se da desde dos aspectos principales, el primero encaminado hacia contextos referentes a la parte educativa y el segundo se abarca desde el punto de vista de las tecnologías de información.

Dada la diversidad de aspectos que se pueden tener en cuenta y teniendo como referente estudios realizados en diferentes partes del mundo, así como los enfoques que buscan solucionar problemas diversos de aprendizaje, se identifican características que permitan lograr un acercamiento a una clasificación general de aspectos que conduzcan a proponer una estructura general de los ítems que pueden incluirse en un modelo general, de modo que abarque aspectos tecnológicos y, por supuesto, educativos; por lo cual se incluyen estudios realizados desde 2012 a 2017 preferiblemente. Otro aspecto que se tuvo en cuenta está relacionado con el hecho de que la evolución de las tecnologías de la información hace que día a día se incorporen nuevos elementos en los procesos, generando que éstos sean dinámicos y evolucionen de acuerdo con las situaciones que se vayan presentado.

Basado en la definición propuesta y en las experiencias descritas en la literatura técnica sobre el tema de educación ubicua, donde se sustentan diferentes perspectivas, se presenta una clasificación de los diferentes aspectos que se pueden tener en cuenta para implementar un proceso de formación U-learning.

\section{ÁREAS DEL CONOCIMIENTO QUE PUEDE APOYAR}

Partiendo de estudios realizados por diferentes investigadores en varias partes del mundo y, dado el desarrollo que está teniendo este tipo de herramientas para el apoyo a los procesos de enseñanza/aprendizaje, se podría afirmar que casi en cualquier área del conocimiento puede ser implementada. A continuación, se mencionan algunos estudios que han desarrollado investigaciones en esta área.

Vázquez (2014) presenta un proyecto desarrollado para apoyar la enseñanza de los conceptos de Microeconomía, dirigida a estudiantes de una Escuela Politécnica. Este proyecto se basó en la implementación de una aplicación para dispositivos móviles ( $a p p$ ), en la cual se establecieron unos objetivos claros de aprendizaje, los cuales se busca cumplir con el desarrollo de la app. La app está diseñada para ser 
utilizada de dos maneras principales: como recurso para el autoaprendizaje o como herramienta para complementar lo explicado por el docente.

En el caso de la enseñanza de las matemáticas, se han implementado diferentes proyectos, uno de ellos es el presentado en Villa Martínez, Tapia Moreno y López Miranda (2010) quienes, para el diseño de la interfaz, toman como referencia las redes bayesianas, las cuales "han probado ser populares en aplicaciones de inteligencia artificial por su capacidad de representar relaciones causales y de razonar con incertidumbre" (Villa Martínez, Tapia Moreno y López Miranda, 2010, p. 129).

Otra aplicación en el caso del área de las matemáticas es la expuesta en Huang, $\mathrm{Wu}$, Chen, Yang y Huang (2012). Allí crearon un ambiente que le permite a estudiantes de segundo año de primaria aprender el tema de suma y resta, mediante la implementación de simulaciones de tiendas virtuales; de esta forma se busca disminuir el temor de los estudiantes por la asignatura y motivar el aprendizaje de la misma.

En diferentes niveles educativos hay una preocupación constante por apoyar los procesos de aprendizaje de una segunda lengua, especialmente el idioma inglés, por lo cual se han desarrollado proyectos enfocados con la finalidad de fortalecer esta habilidad, tal como lo expone Lin, Fulford, Ho, Lyoda y Ackerman (2012), donde proponen el apoyo del proceso de aprendizaje de estudiantes que estén adelantando actividades para adquirir competencias en inglés como segunda lengua, mediante el uso de un dispositivo mp3 que les permite escuchar música y leer la letra de las canciones. Con este fin desarrollaron una aplicación que permite realizar preguntas sobre la música que escuchan con el fin de analizar el uso correcto del sustantivo y el verbo.

García-Sánchez (2016) presenta un caso de estudio sobre el apoyo al proceso de formación en segunda lengua mediante la implementación de actividades de aprendizaje, actividades evaluativas mediante el uso de las TIC, apoyándose en recursos tales como videos para la explicación y la presentación de evaluaciones. Este caso de estudio presenta resultados favorables en la mejora de las competencias comunicativas de los participantes que apoyaron su proceso de aprendizaje con las herramientas planteadas.

Así mismo, se diseñó un sistema de aprendizaje ubicuo para la enseñanza de geografía., El proyecto consistió en el desarrollo e implementación de un sistema de aprendizaje de geografía ubicuo, el cual cuenta con 120 elementos de aprendizaje (4 temas y 30 elementos por cada tema), fue probado en una escuela del sur de Taiwan, donde se definieron dos grupos: uno experimental y otro de control, ambos grupos contaban con el mismo maestro. Los estudiantes que tenían acceso a la aplicación podían navegar por los diferentes temas, y podían presentar actividades de evaluación. Como conclusión de la investigación, los estudiantes del grupo experimental obtuvieron mejores calificaciones en el examen final que los estudiantes del grupo de control (Yang y Chang, 2016). 
Las áreas de aplicación pueden llegar a ser tan diversas que Martin y Ertzberger (2013) presentan un estudio en el cual se explora el uso de dispositivos móviles para el aprendizaje de contenido artístico situado en el contexto.

Un caso de cómo se puede llegar a implementar la educación ubicua para la enseñanza de algunos conceptos de física en el proceso de aprendizaje de los fundamentos del péndulo en una escuela secundaria, se presenta en Purba y Hwang (2017).

De acuerdo con los estudios descritos en este apartado, se puede analizar que la aplicación de la educación ubicua puede darse en diferentes contextos y diferentes áreas del conocimiento.

En la figura 3 se presenta una síntesis de las áreas en las cuales se puede llegar a usar U-learning.

Figura 3. Áreas de Conocimiento donde se puede aplicar el aprendizaje ubicuo

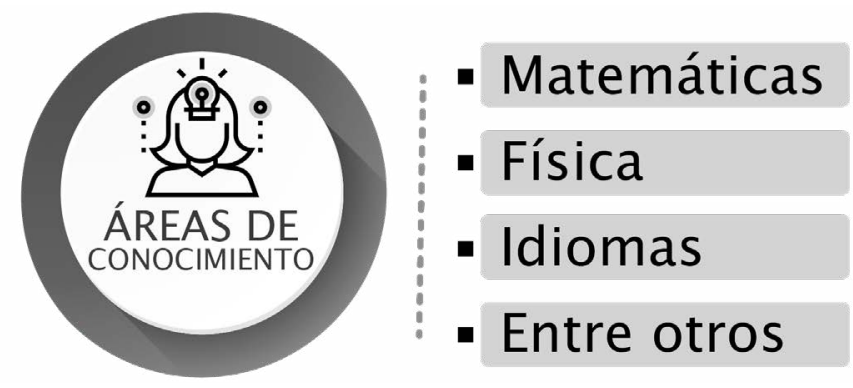

\section{NIVELES EDUCATIVOS EN LOS QUE SE PUEDE IMPLEMENTAR}

Dada la aplicabilidad que tiene este tipo de aprendizaje, se encuentra que es viable su implementación tanto para educación formal como para educación informal. En cuanto al nivel de educación formal se han implementado proyectos a nivel de la escuela Politécnica como es el caso de Vázquez (2014).

En los niveles educativos de secundaria y primaria también se han desarrollado aplicaciones que permiten su implementación como es el caso de Tarng y Ou (2012). En este proyecto se realizó un estudio sobre el diseño de un ambiente virtual de aprendizaje, con el fin de que estudiantes de ecología de escuelas de primaria y secundaria pudiesen aprender el proceso de creación y gestión de un jardín de mariposas.

A nivel de primaria se han trabajado proyectos como el planteado en Huang et al. (2012). Los investigadores crearon un ambiente que le permita a estudiantes de segundo año de educación primaria aprender el tema de suma y resta. 
A nivel de educación informal Liu, Ogata y Mouri (2015) desarrollaron una aplicación para enseñar japonés a estudiantes internacionales mediante el uso de redes sociales.

También hay que analizar la perspectiva del docente. Lin et al. (2012) plantea un estudio piloto donde se analizan aspectos de los docentes, tales como actitud, creencias, concepción del proceso de enseñanza, concepción del proceso de aprendizaje, uso de la tecnología móvil. El estudio revela que se manifiesta un cambio positivo por parte de los docentes en la adopción de las tecnologías móviles como apoyo al proceso de aprendizaje de los estudiantes.

El apoyo de un mentor o tutor en estos procesos es un aspecto fundamental, dado que se trata de implementar un aprendizaje situado basado en el contexto. Joo, Park y Choi (2014) presentan la implementación de un modelo de enseñanza-aprendizaje mediante el desarrollo de un Sistema de Gestión de Aprendizaje (LMS por sus siglas en inglés) donde se establece el nivel y objetivo de aprendizaje de cada participante.

En general, se plantea la implementación de aulas del futuro, donde incorporen el trabajo colaborativo a través de la aplicación de procesos que sean enriquecidos por todos los participantes, siendo mediado con el uso de recursos, tales como videoconferencia, diccionario de términos en línea y fuera de línea, contenido adaptado a las necesidades (Marinagi, Skourlas y Belsis, 2013), entre otros recursos.

La figura 4 presenta una síntesis de los niveles de formación en los cuales se puede aplicar el aprendizaje ubicuo.

Figura 4. Niveles de formación

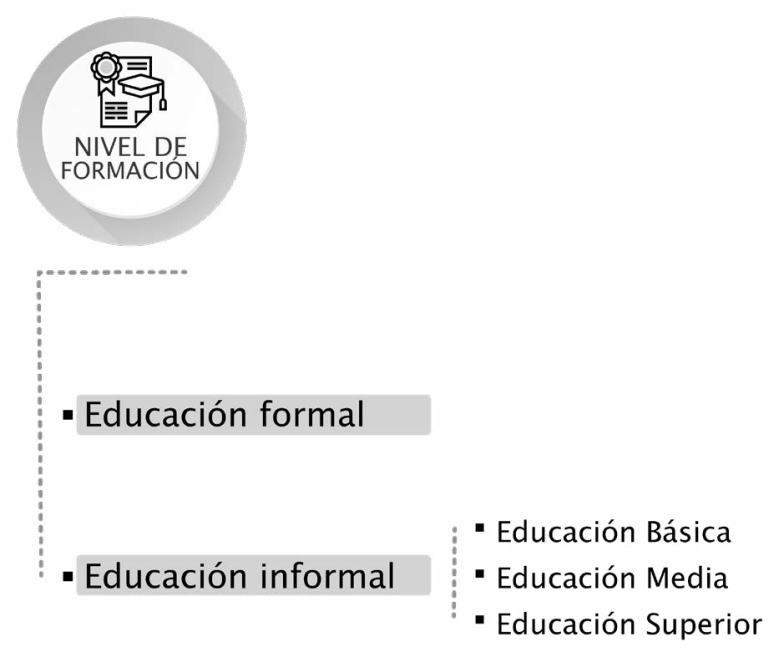




\section{TECNOLOGÍAS DE LA INFORMACIÓN QUE PERMITEN SU IMPLEMENTACIÓN}

Dentro de las características mencionadas para la implementación de la educación ubicua como apoyo al proceso de aprendizaje, se deben tener en cuenta los aspectos tecnológicos: por un lado, lo referente a la infraestructura y, por el otro, lo referente al software (Villa Martínez et al., 2010). En el estudio realizado por Lai y Mao (2014), los autores analizan el uso de los dispositivos móviles como parte del proceso de aprendizaje en las universidades chinas. Se encuentra que es necesario que la institución académica permita el acceso a las redes wi-fi por parte de los estudiantes, dado que el uso de datos para estos dispositivos puede generar costos adicionales. El estudio arrojó además que los estudiantes sienten más motivación por el uso de estos recursos en el proceso enseñanza-aprendizaje.

Utilizar recursos de tecnologías de la información como los servicios web puede ayudar a garantizar la interoperabilidad de las aplicaciones y, por consiguiente, su mayor uso, tal es el caso presentado por Chi, Kuo y Lin (2012). En este proyecto implementaron una base de datos para almacenar música dentro de un proceso de apoyo al aprendizaje del idioma inglés.

Un aspecto que se puede aprovechar es el apoyo de estos procesos mediante el uso de herramientas ya implementadas, como es el caso de las redes sociales (Liu et al., 2015), las cuales pueden ser aprovechadas para el envío de información no solo a los estudiantes, sino a los padres (Reis, Escudeiro y Escudeiro, 2012). Otro recurso que puede ser aprovechado es el uso de videos (García-Sánchez, 2016). Un avance relevante se encuentra en Atif, Mathew y Lakas (2014), donde presentan la creación de un campus inteligente para soportar educación ubica. Allí plantean diferentes aspectos que se puede complementar en los espacios físicos de aprendizaje con servicios digitales y sociales.

Diseñar ambientes virtuales que se apoyen en el uso de teléfonos inteligentes conectados a redes $3 \mathrm{G}$ y sistemas de ubicación GPS, para conocer la ubicación del usuario y enviarle información referente al sitio en el que se encuentra, es lo planteado en Tarng y Ou (2012). También se pueden aprovechar dispositivos tecnológicos ya desarrollados como, por ejemplo, un dispositivo mp3 (Lin, 2012), como se mencionó anteriormente. Otro dispositivo que se puede aprovechar son las tabletas. Huang et al. (2012) desarrollaron un software para estos dispositivos con Sistema Operativo Windows 7, en el cual crearon dos ambientes de aprendizaje para el tema de las restas en matemáticas, uno dentro del aula de clase y otro fuera de ella, buscando aplicar en diferentes contextos en los que se desenvuelve el estudiante.

Una tecnología usada en la implementación de algunos proyectos es Cloud Computing, la cual consiste en guardar los datos necesarios para el uso de las aplicaciones móviles enfocadas en la enseñanza en la nube computacional. De esta forma se solucionan problemas como falta de memoria o baja capacidad de procesamiento (Bayonet Robles y Patino Matos, 2014). Yu y Yang (2015) 
proponen crear una célula de aprendizaje donde el almacenamiento de los recursos desarrollados se realice en la nube. Así mismo, Khan, Othman, Khan, Abid y Madani (2015) implementaron el proyecto llamado MobiByte, el cual también usa la nube como infraestructura tecnológica.

Un hecho significativo es la posibilidad de desarrollar aplicaciones para dispositivos móviles que se adapten a los diferentes tamaños y tecnologías de los dispositivos (Mercurio, Torre y Torsani, 2014; Velev, 2014) como, por ejemplo, en Li, Ogata, Hou y Ackerman (2012), quienes proponen un sistema que se adapta no solo a los dispositivos que se están usando sino que lleva un registro del proceso de aprendizaje.

También se debe pensar que un objetivo de la educación ubicua es mejorar la experiencia con el usuario a través de la interacción, por lo cual Chiou, Tseng \& Hsu (2014) proponen un algoritmo combinado de navegación B-MON, generando mejores resultados en el proceso de aprendizaje. Otro objetivo es crear interfaces humanas más atractivas e intuitivas para los usuarios y, de este modo, enriquecer el proceso de interacción con contenidos educativos (Kaneko, Nakamura, Okada y Matsuguma, 2012).

Partiendo del hecho que el aprendizaje ubicuo permitirá tener registros digitales de los procesos desarrollados por cada aprendiz, se crea una nueva oportunidad para poder analizar dichos datos y sobre ellos proponer aspectos que mejoren continuamente dichos procesos (Ogata y Mouri, 2015).

Se debe partir de la realidad que se desarrolla y vive en cada comunidad, por lo cual se hace necesario identificar las competencias desarrolladas en el uso de los dispositivos móviles con el fin de hacer un mejor aprovechamiento del uso de internet en la educación y evitar los efectos negativos de este. En un estudio realizado en India, Pandey (2014) realiza un análisis sobre la necesidad de incluir en el currículo de los estudiantes actividades enfocadas a desarrollar competencias en el uso de las tecnologías de información.

Para la incorporación de las TIC en el proceso de aprendizaje, se hace necesario la capacitación en su uso tanto a estudiantes como a docentes (Chatterjee y Nath, 2015). En cuanto al diseño de la interfaz, debe ser definida de acuerdo con las habilidades tecnológicas del estudiante (Villa Martínez et al., 2010).

En el estudio presentado por Dankasa (2014), se realiza un análisis de las condiciones particulares que se encuentran en África, particularmente con el acceso y disponibilidad de redes inalámbricas de datos y el costo que ello implica, con el fin de crear aplicaciones que puedan ser usadas fuera de línea.

Como se puede observar, es necesario tener en cuenta componentes tecnológicos que desarrollen una interacción exitosa por parte del usuario final, con el fin de generar procesos efectivos de aprendizaje. Adicionalmente, la infraestructura tecnológica sobre la cual se implemente la solución debe garantizar niveles óptimos de calidad, con el fin de ofrecer satisfacción al usuario.

De acuerdo con los aspectos mencionados, la figura 5 presenta una síntesis de lo expuesto en este apartado. 
Figura 5. Aspectos tecnológicos en educación ubicua

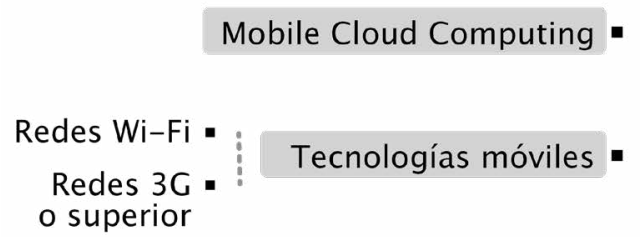

Infraestructura

Ambientes de enseñanza

Micrositios web

Aplicaciones móviles

Uso de herramientas ya desarrolladas

Redes sociales .

Videos .

Dispositivos

Tablets -

MP3

Celulares Inteligentes .

\section{MODELOS DE APRENDIZAJE}

Dada la diversidad de formas de aprendizaje y la necesidad de tener en cuenta el contexto en el cual se están desarrollando dichos procesos, se han planteado proyectos con diferentes modelos de aprendizaje. Así mismo, partiendo del hecho que el aprendizaje se relaciona con un aspecto muy importante como la evaluación, se hace necesario plantear y analizar nuevos contextos para dicho proceso dado que, al cambiar la forma de aprender, también se deben modificar las estrategias de aprendizaje, tal como lo proponen BenSassi, Laroussi y BenGhezela (2015). 
Phumeechanya y Wannapiroon (2013) realizan un planteamiento de actividades para un aprendizaje escalonado en un ambiente ubicuo, mediante la estrategia de resolución de problemas, en el cual incluye un módulo que permita evaluar el avance del proceso de aprendizaje.

Los resultados de la investigación fueron los siguientes: 1) Las actividades de aprendizaje contaban con cuatro componentes: a) estudio del contenido, b) presentar el problema, c) problema de planificación de la solución, d) resolución de problemas, e) identificación de la generalización y principios derivados de estudiar el problema. El objetivo de las actividades de aprendizaje es el desarrollo de habilidades para resolver problemas. 2) Los expertos coinciden en que unas actividades de aprendizaje eran apropiadas en un buen nivel.

Suresh Babu, Vijay Daniel y Joshna (2015) plantean un modelo de aprendizaje ubicuo basado en problemas para apoyar el proceso de enseñanza del tratamiento de imágenes digitales. Vázquez (2014) plantea un modelo basado en un enfoque activo y exploratorio en un entorno multimedia. Así mismo, Reis et al. (2012) desarrolló una aplicación para enviar comunicaciones tanto a estudiantes como padres, dentro de un proceso de acompañamiento continuo.

El aprendizaje basado en juegos se expone en los trabajos de Tarng y Ou (2012) y Huang et al. (2012). En este último caso se crea un modelo instruccional basado en el concepto de tiendas virtuales.

Otro modelo de aprendizaje es el adaptativo, en el cual se tendrán en cuenta los conocimientos previos del estudiante, con el fin de ajustar los contenidos de aprendizaje a las características propias del mismo (Villa Martínez et al., 2010).

Los mapas mentales han sido una herramienta de uso frecuente dentro de los procesos de enseñanza aprendizaje. A este respecto, Hwang, Wu, Huang, y Kuo (2012) proponen un modelo de aprendizaje teniendo como estrategia el desarrollo de mapas mentales mediante el uso de un dispositivo móvil, en el cual se instala el software necesario para el desarrollo de la actividad, en un curso de planificación de negocios. El estudio se aplicó a 90 estudiantes divididos en dos grupos, con el fin de tener el grupo de prueba y el grupo de control. Los resultados del estudio demostraron que los estudiantes del grupo experimental pudieron desarrollar más su creatividad por medio del uso del dispositivo móvil, contrario a lo que ocurrió con los estudiantes que aprendieron el tema de manera tradicional.

Teniendo en cuenta que existen diferentes estilos de aprendizaje, Caceffo, Rocha y Azevedo (2015) proponen un modelo de aprendizaje activo orientado a que los estudiantes participen activamente junto con su profesor en el proceso de aprendizaje, el cual también tiene en cuenta los estilos propios de aprendizaje.

Dado que los procesos de aprendizaje surgen también de la interacción con otras personas, Temdee (2016) propone un modelo inicial en el que mediante agentes se simula como puede llegar a implementarse esa interacción dentro de un entorno de aprendizaje ubicuo. 
La figura 6 presenta una síntesis de lo expuesto en este apartado.

Figura 6. Modelos de Aprendizaje

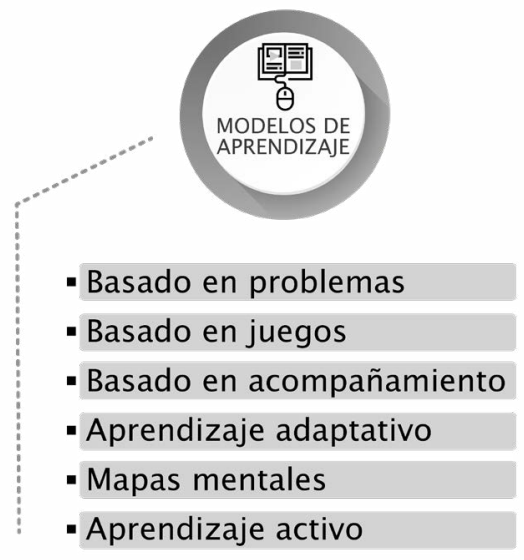

\section{Caso destacable}

Es importante hacer un énfasis especial al proyecto que se está desarrollando en España, con el apoyo de la Fundación Telefónica, el cual consiste en la creación del "Laboratorio Social de Mobile Learning", y busca implementar experiencias de diferentes partes del mundo relacionadas con el aprendizaje por medio de dispositivos móviles (Fundación Telefónica, 2014). Dentro de los diferentes proyectos que han desarrollado, se destaca un proyecto que buscó involucrar en el proceso las habilidades que ya poseen los estudiantes de 12 años en el uso de los teléfonos inteligentes para ser aprovechadas en los procesos de aprendizaje.

\section{CONCLUSIONES}

De acuerdo con los proyectos analizados, se puede evidenciar que $U$-learning es una tendencia a nivel mundial en la cual se busca llevar el aprendizaje a contextos fuera de los tradicionales, apoyando estos procesos en análisis y situaciones de la vida real para buscar que el aprendizaje sea más efectivo y se adquiera de diferentes formas.

Se encontró que las herramientas informáticas desarrolladas permiten ser utilizadas directamente en los dispositivos móviles o a través de conexiones a redes inalámbricas para poder acceder a dichos recursos, haciendo necesario un análisis del tipo de dispositivo en el cual se van a desplegar para poder garantizar su uso. 
La educación ubicua es un concepto en desarrollo, que todavía está en exploración, en el cual se pueden llegar a hacer aportes importantes que permitan una adecuada incorporación en los procesos de aprendizaje de los dispositivos móviles.

Estudios realizados sobre la mejora de los procesos de aprendizaje han demostrado, por ejemplo, que la implementación de herramientas computacionales en la educación aquí y ahora sobre la educación instruccional dada por una computadora mejoran positivamente el proceso de aprendizaje (Martin y Ertzberger, 2013; Tutty y Martin, 2014; Martin y Ertzberger, 2015). Particularmente, un estudio de caso desarrollado en Korea a 30 estudiantes universitarios por medio de una aplicación desarrollada en App Inventor generó como conclusiones que los estudiantes tuvieron diferencias significativas en cuatro factores de motivación: atención, relevancia, constancia y satisfacción (Bae y Lee, 2015).

En la figura 7, se presenta el compendio de lo presentado en los apartados anteriores. 
Figura 7. Elementos de aprendizaje ubicuo

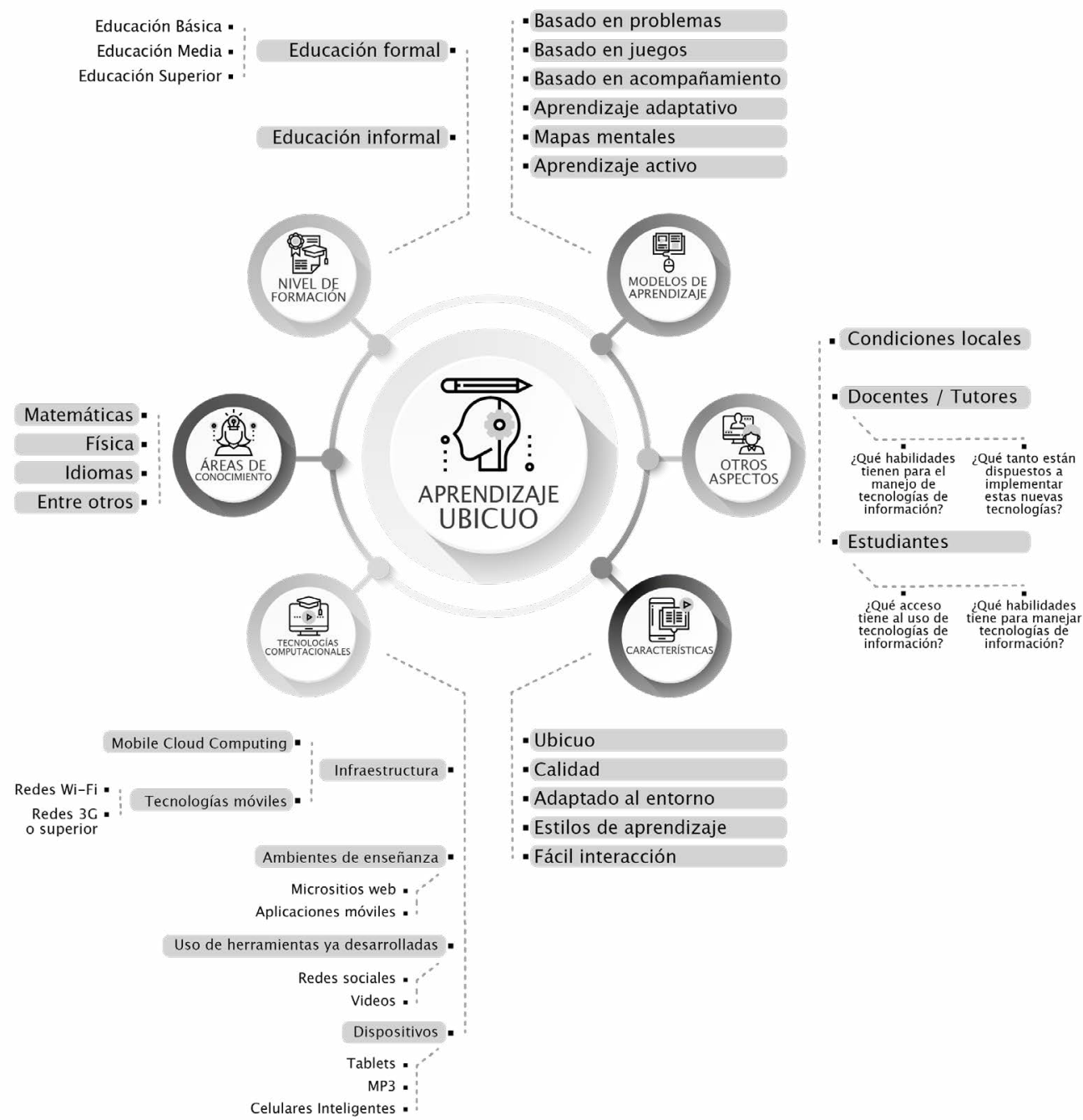

La figura presenta diferentes aspectos que pueden ser tenidos en consideración para desarrollar ambientes ubicuos enriquecidos por el contexto y que mejoren la experiencia del estudiante mediante la mediación de tutores y/u otras herramientas de apoyo. Es necesario aclarar que lo presentado no es una lista exhaustiva de los aspectos que pueden ser tenidos en cuenta, son algunos de los elementos tomados del análisis de estado de arte realizado. 
De acuerdo con el presente análisis, se puede evidenciar que existe una evolución acerca de la forma de implementar herramientas ubicuas para el apoyo a los procesos de enseñanza/aprendizaje. Tal es el caso de los proyectos en los cuales se han desarrollado algunas herramientas informáticas, así como la implementación de escenarios completos de aprendizaje ubicuo. Es importante resaltar que la incorporación de este aprendizaje depende mucho de la evolución en la inclusión de dispositivos móviles en dichos procesos.

La apropiación de procesos de educación ubicua plantea la necesidad de abordarla desde todas las perspectivas, tales como la tecnología, el ambiente de aprendizaje, las metodologías, los ambientes físicos de implementación, entre otros (Hwang, 2014; Atif et al., 2015).

\section{REFERENCIAS}

Atif, Y., Mathew, S., y Lakas, A. (2014). Building a smart campus to support ubiquitous learning. Journal of Ambient Intelligence and Humanized Computing, 6(2), 223-238. doi:10.1007/s12652-0140226-y

Bae, J., y Lee, H. (2015). Development of Learner-Centric Teaching-Learning Application Model for Ubiquitous Learning. Advances in Computer Science and Ubiquitous Computing, 373, 353359. doi: https://doi.org/10.1007/978981-10-0281-6 51

Barragán Sánchez, R., Mimbrero Mallado, C., y Pacheco González-Piñal, R. (2013). Cambios pedagógicos y sociales en el uso de las TIC U-learning y U-portafolio. Revista Electrónica de Investigación y Docencia (REID), 10, 7-20. Recuperado de https://revistaselectronicas.ujaen.es/ index.php/reid/article/view/989/816

Bayonet Robles, L., y Patino Matos, A. (2014). Mobile Cloud aplicado en las Escuelas Rurales de República Dominicana, 11. Recuperado de http://www.academia. edu/7563165/Mobile Cloud aplicado en las Escuelas Rurales de Republica Dominicana

BenSassi, M., Laroussi, M., y BenGhezela, H. (2015). Evaluation Framework for the Dependability of Ubiquitous Learning
Environment. Computer and Information Science, 39-55. doi: 10.1007/978-3-31910509-3_4.

Burbules, N. C. (2012). El aprendizaje ubicuo y el futuro de la enseñanza. Encounters/ Encuentros/Rencontres on Education, 13, 3-14. Recuperado de https://ojs.library. queensu.ca/index.php/encounters/\%20 article/view/4472/4513

Burbules, N. C. (2014). Los significados de "aprendizaje ubicuo". Education Policy Analysis Archives, 22(104), 1-10. Artículo publicado originalmente en Revista de Política Educativa, Año 4, Número 4, UdeSA-Prometeo, Buenos Aires, 2013. doi:10.14507/epaa.v22.1880.

Caceffo, R., Rocha, H., y Azevedo, R. (2015). A Ubiquitous, Pen-Based and Touch Classroom Response System Supported by Learning Styles. Human-Computer Interaction Series, 373-380. doi: https:// doi.org/10.1007/978-3-319-15594-4 39

Cantillo Valero, C. Roura Redondo, M., y Sánchez Palacín, A. (2012). Tendencias actuales en el uso de dispositivos móviles en educación.La Educ@Ción Digital Maganize, 147, 1-21. Recuperado de http://educoas.org/portal/la educacion digital/147/pdf/art unned en.pdf 
Carmona, L., y Puertas. F. (2012). ULearning: la revolución del aprendizaje. Observatorio de Recursos Humanos y Relaciones Laborales, 70, 24-26. Recuperado de https://factorhuma.org/ attachments secure/article/9616/c369 ulearning revolucion aprendizaje.pdf

Chatterjee, P., y Nath, A. (2015). The Future ICT Education in India-A Pilot Study on the Vision of Ubiquitous Learning in Higher Education. Advances in Intelligent Systems and Computing, 340, 599-609. doi: 10.1007/978-81-322-2247-7_61

Chi, C., Kuo, C., \& Lin, K. (2012). A Design of Mobile Application for English Learning. 2012 IEEE Seventh International Conference On Wireless, Mobile And Ubiquitous Technology In Education. doi: 10.1109/wmute.2012.59.

Chiou, C. K., Tseng, J. C. R., y Hsu, T. Y. (2014). Navigation Mechanism in Blended Context-Aware Ubiquitous Learning Environment. Multimedia and Ubiquitous Engineering, 308, 199-204. doi: 10.1007/978-3-642-54900-7_29.

Dankasa, J. (2014). Examining the progression of mobile technologies and their applications to learning environment: Implications for m-learning in Africa. International Journal of Research Studies in Educational Technology, 1-14. doi: 10.5861/ijrset.2014.825.

Fidalgo, Á. (2013). ¿Qué es el aprendizaje ubicuo?. Blog de Ángel Fidalgo para reflexionar sobre innovación educativa. Recuperado de https:// innovacioneducativa.wordpress. com/2013/05/13/que-es-el-aprendizajeubicuo/

Fundación Telefónica (2014). La experiencia del Laboratorio Mobile Learning [Ebook] (pp. 4-6). Recuperado de https://publiadmin.fundaciontelefonica.com/media/publicaciones/268/ laboratorio mobile learning.pdf? $\mathrm{ga}=2.223818204 .693212902 .1538171306-$ 1348322291.1538083636
García-Sánchez, S. (2016). Educación a distancia, interactiva y ubicua para el aprendizaje de lengua inglesa. Revista Academia y Virtualidad, 9(1), 66-88. doi: http://dx.doi.org/10.18359/ravi.1706

Gros, B. (2015). La caída de los muros del conocimiento en la sociedad digital y las pedagogías emergentes. Education in the Knowledge Society (EKS), 16(1), 58-68. doi: 10.14201/eks20151615868.

Huang, S., Wu, T., Chen, H., Yang, P., y Huang, Y. (2012). Mathematics assisted instruction system of M/U-Learning environment. 2012 IEEE Seventh International Conference on Wireless, Mobile and Ubiquitous Technology in Education, 301-305. doi: 10.1109/ WMUTE.2012.72.

Hwang, G. (2014). Definition, framework and research issues of smart learning environments - a context-aware ubiquitous learning perspective. Smart Learning Environments, 1(4), 1-14. doi: 10.1186/s40561-014-0004-5.

Hwang, G. Wu, C., Huang, I., y Kuo, F. (2012). A Mind Map-oriented Mobile Learning Approach to Promoting Creative Thinking Ability of Students in a Business course. 2012 IEEE Seventh International Conference on Wireless, Mobile and Ubiquitous Technology in Education, 2012 IEEE, 7. doi: 10.1109/wmute.2012.60.

Joo, K., Park, N., y Choi, J. (2014). An Adaptive Teaching and Learning System for Efficient Ubiquitous Learning. Ubiquitous Information Technologies and Applications, 28o, 659-666. doi: https:// doi.org/10.1007/978-3-642-41671-2 84

Kaneko, K., Nakamura, T., Okada, Y., y Matsuguma, H. (2012). Open Device Control (OpenDC) Human Interface Device Framework for Interactive Applications Including Educational Contents in Ubiquitous Environments. 2012 IEEE Seventh International Conference on Wireless, Mobile and 
Ubiquitous Technology in Education, 8. doi: 10.1109/WMUTE.2012.29.

Khan, A. R., Othman, M., Khan, A. N., Abid, S. A., y Madani, S. A. (2015). MobiByte: An Application Development Model for Mobile Cloud Computing. Journal of Grid Computing, 13(4), 605-628. doi: 10.1007/ s10723-015-9335-x.

Kinshuk. (2015). Roadmap for Adaptive and Personalized Learning in Ubiquitous Environments. Ubiquitous learning enviroments and technologies, 1-13. doi: 10.1007/978-3-662-44659-1_1.

Lai, D. \& Mao, C. (2014). A Study on factors affecting the mobile learning of undergraduate students in China. Creative Education, 5, 372-375. doi: 10.4236/ce.2014.55046.

Li, M., Ogata, H., Hou, B., y Uosaki, N. (2012). Context-aware multimodal interfaces enhancing ubiquitous learning, 2. Recuperado de http://ceur-ws.org/Vol955/papers/paper 25.pdf

Lin, M., Fulford, C., Ho, C., Lyoda, R., y Ackerman, L. (2012). Possibilities and Challenges in Mobile Learning for $\mathrm{K}-12$ Teachers: A Pilot Retrospetive Survey Study. 2012 IEEE Seventh International Conference on Wireless, Mobile and Ubiquitous Technology in Education. doi: 10.1109/WMUTE.2012.31.

Liu, S., Ogata, H., y Mouri, K. (2015). Accelerate location-based context learning for second language learning using ubiquitous learning Log. Emerging Issues In Smart Learning, 53-60. doi: 10.1007/978-3-662-44188-6_7.

Marinagi, C., Skourlas, C., y Belsis, P. (2013). Employing Ubiquitous Computing Devices and Technologies in the Higher Education Classroom of the Future. Procedia - Social And Behavioral Sciences, 73, 487-494. doi: 10.1016/j.sbspro.2013.02.081.

Martin, F., y Ertzberger, J. (2013). Here and now mobile learning: An experimental study on the use of mobile technology. Computers \& Education, 68, 76-
85. doi: http://dx.doi.org/10.1016/j. compedu.2013.04.021

Martin, F., y Ertzberger, J. (2015). Effects of reflection type in the here and now mobile learning environment. British Journal Of Educational Technology, 47(5), 932-944. doi: 10.1111/bjet.12327.

Mercurio, M., Torre, I., y Torsani, S. (2014). Responsive Web and Adaptive Web for Open and Ubiquitous Learning. Open Learning And Teaching In Educational Communities, 8719, 452-457. doi: 10.1007/978-3-319-11200-8_41.

Ogata, H., y Mouri, K. (2015). Connecting Dots for Ubiquitous Learning Analytics. Hybrid Learning: Innovation in Educational Practices. ICHL 2015. Lecture Notes in Computer Science, 9176, 46-56. doi: 10.1007/978-3-319-206219_4.

Pandey, R. (2014). Digital Citizenship \& Internet Maturity in Schools of India. Recuperado de http://www.wise-qatar. org/edhub/digital-citizenship-internetmaturity-schools-india? ga=1.55743659. 189139681.1491558830

Phumeechanya, N.,yWannapiroon, P.(2014). Design of problem-based with scaffolding learning activities in ubiquitous learning environment to develop problem-solveing skills. Procedia Social and Behavioral Sciences, 116, 4803-4808. doi: 10.1016/j. sbspro.2014.01.1028.

Purba, S. W. D., y Hwang, WY. J. (2017). Investigation of Learning Behaviors and Achievement of Vocational High School Students Using an Ubiquitous Physics Tablet PC App. Journal of Science Education and Technology, 26(3), 322331. doi: 10.1007/s10956-016-9681-x.

Reis, R., Escudeiro, P., y Escudeiro, N. (2012). Educational Resources for Mobile Wireless Devices: A Case Study. 2012 IEEE Seventh International Conference on Wireless, Mobile and Ubiquitous Technology in Education, 4. doi: 10.1109/ WMUTE.2012.64. 
Suresh Babu, C. V., Vijay Daniel, J., y Joshna, S. (2015). Innovative method of teaching digital signal processing using ubiquitous learning strategies. Proceedings of the International Conference on Transformations in Engineering Education, 323-335. doi:.10.1007/97881-322-1931-6_38.

Tarng, W., y Ou, K.-L. (2012). A Study of Campus Butterfly Ecology Learning System based on Augmented Reality and Mobile Learning. 2012 IEEE Seventh International Conference on Wireless, Mobile and Ubiquitous Technology in Education, 5. doi:10.1109/ WMUTE.2012.17.

Temdee, P. (2016). Agent-based modeling of collaborative interaction in ubiquitous learning environment using local dynamic behavior. Artificial Life and Robotics, 21(2), 215-220. doi: 10.1007/s10015-0150256-3.

Tutty, J., y Martin, F. (2014). Effects of practice type in the here and now mobile learning environment. I-Manager's Journal of Educational Technology, 11(2), 17-27. Recuperado de https://files. eric.ed.gov/fulltext/EJ1098535.pdf

Vázquez, E. (2014). Mobile Distance Learning with Smartphones and Apps in Higher Education. Educational Sciences: Theory \& Practice. 14 (4), 1505-1520. doi: 10.12738/estp.2014.4.2012.

Velev, D. (2014). Challenges and Opportunities of Cloud-Based Mobile Learning. International Journal of Information and Education Technology, 4(1), 49-53. doi: 10.7763/ijiet.2014. $\mathrm{v} 4.367$.
Villa Martínez, H., Tapia Moreno, F. \& López Miranda, C. (2010). Aprendizaje Ubicuo En La Ensenanza de Las Matematicas. Revista Estudios Culturales, 3(5), 123-136. Recuperado de https://dialnet.unirioja. es/servlet/articulo?codigo $=3739983$

Yahya, S., Arniza, E., \& Jalil, K. A. (2010). The definition and characteristics of ubiquitous learning: A discussion. International Journal of Education and Development using Information and Communication Technology (IJEDICT), 6(1), 1-11. Recuperado de https://search. proquest.com/docview/237249181/fulltextPDF/4462855C726E44B6PQ/1?accountid $=43592$

Yang, H. C., y Chang, WC. (2017). Ubiquitous smartphone platform for K-7 students learning geography in Taiwan. Multimedia Tools and Applications, 76(9), 1165111668. doi:10.1007/s11042-016-3325-2.

Yu, S., y Yang, X. (2015). A Resource Organization Model for Ubiquitous Learning in a Seamless Learning Space. Seamless Learning in the Age of Mobile Connectivity, 141-158. doi:10.1007/978981-287-113-8_8.

Zapata-Ros, M. (2012). Calidad en entornos ubicuos de aprendizaje. Revista de Educación a Distancia, 9(31), 1-12. Recuperado de https://www.um.es/ead/ red/31/zapata ros.pdf

Zapata-Ros, M. (2015). Teorías y modelos sobre el aprendizaje en entornos conectado y ubicuos. Bases para un nuevo modelo teórico a partir de una visión crítica del "conectivismo". Education in the Knowledge Society (EKS), 16(1), 69102. doi:10.14201/eks201516169102. 


\section{PERFIL ACADÉMICO Y PROFESIONAL DE LOS AUTORES}

Carmen Inés Báez Pérez es Ingeniera de Sistemas, Especialista en Telemática, $\mathrm{MsC}$ en Ciencias de la Información, Candidata a doctora en Ingeniería de Proyectos, de la Universidad Tecnológica de Panamá. Con publicaciones en revista indexada en el área de Sistemas Distribuidos. Publicación del libro: "Proceso de desarrollo de software: basado en la articulación de RUP y CMMI priorizando su calidad" y del libro "Redes Inalámbricas de sensores en ambiente Grid". Docente titular de la Universidad de Boyacá.

E-mail: cibaez@uniboyaca.edu.co

Dirección:

Facultad de Ciencias e Ingeniería

Universidad de Boyacá

Programa de Ingeniería de Sistemas

Cra 2 Este No. 64-169

Tunja (Colombia)

Clifton Eduardo Clunie Beaufond es Licenciado en Ingeniería de Sistemas Computacionales en el Instituto Politécnico de la Universidad de Panamá, Maestría y Doctorado en Ingeniería de Sistemas y Computación en la Universidad Federal de Rio de Janeiro - Brasil, Estudios Doctorales en Tecnología Educativa en la Universidad de Ihleas Baleares - España. Ha sido Asesor en Trabajos de Graduación a nivel de Licenciatura e Ingeniería y Asesor de Tesis en los programas de Maestría y Doctorado.

E-mail: clifton.clunie@utp.ac.pa

Dirección:

Universidad Tecnológica de Panamá

Avenida Universidad Tecnológica de Panamá, Vía Puente Centenario,

Campus Metropolitano Víctor Levi Sasso.

Ciudad de Panamá, Panamá

Fecha de recepción del artículo: 09/08/2018

Fecha de aceptación del artículo: 24/09/2018

Fecha de aprobación para maquetación: 03/12/2018 